\begin{tabular}{lll} 
Universidade do Minho & Universidade do Minho \\
Escola de Ciências & $\begin{array}{l}\text { Universidade do Minho } \\
\text { Escola de Ciências }\end{array}$ \\
Centro de Matemática & Departamento de Matemática e Aplicações \\
\hline
\end{tabular}

\title{
Basins of attraction for a quadratic coquaternionic map
}

\author{
M. Irene Falcão ${ }^{a, c} \quad$ Fernando Miranda $^{a, c} \quad$ Ricardo Severino $^{c} \quad$ M. Joana Soares ${ }^{b, c}$
}

a CMAT - Centre of Mathematics, University of Minho, Portugal

${ }^{b}$ NIPE - Economic Policies Research Unit, University of Minho, Portugal

${ }^{c}$ Department of Mathematics and Applications, University of Minho, Portugal

\section{Information}

Keywords:

Iteration of maps,

coquaternions,

attracting cycles,

basins of attraction.

Original publication:

Chaos, Solitons and Fractals 104

(2017) 716-724

DOI:10.1016/j.chaos.2017.09.016

\begin{abstract}
In this paper we consider the extension, to the algebra of coquaternions, of a complex quadratic map with a real super-attractive 8-cycle. We establish that, in addition to the real cycle, this new map has sets of non-isolated periodic points of period 8, forming four attractive 8cycles. Here, the cycles are to be interpreted as cycles of sets and an appropriate notion of attractivity is used. Some characteristics of the basins of attraction of the five attracting 8-cycles are discussed and plots revealing the intertwined nature of these basins are shown.
\end{abstract}

\section{Introduction}

Although lab measurements produce real numbers, history of quantitative science shows that extensions of these numbers have played an essential role in the development of mathematical models, as soon as it became clear that these new numbers evidence an ability to capture the symmetry of a physical system. One such set of numbers, in particular, - the quaternions, introduced in 1843 by the Irish mathematician William Rowan Hamilton (1805-1865) [19] — have become a powerful tool for modeling and solving problems in classical fields of mathematics, engineering and physics [23]. However, quaternions are not the only four dimensional hypercomplex real algebra generalizing complex numbers; for example, one may also consider coquaternions, proposed by James Cockle (1819-1895), [10], at about the same time as quaternions were discovered. Although coquaternions - also known in the literature as split-quaternions or para-quaternions - have received much less attention than quaternions, more recent studies reveal some potential on the use of this mathematical tool. In fact, since the algebra of coquaternions is appropriate to study the $(2+1)$-geometry of 3D Minkowski space, it is no surprise to see different branches of physics - such as the theory of graphene, black holes, quantum gravity, the AdS/CFT correspondence and the gauge theory of gravitation - making use of the richness of coquaternions towards a deeper understanding of the physical phenomena; see [17] and references therein. Some geometrical applications of coquaternions can also be seen in e.g. [2, 21, 27, 28]; a connection between coquaternionic analysis and the four-dimensional quantum field theory is investigated in [14], and in [8] the authors examine the relation between coquaternions and complexified mechanics; for a very recent application of coquaternions in the area of cosmology, see [16]. 
The dynamics of the quadratic map in the complex plane has been object of intense study and can now be considered a well-established theory; see, e.g., $[5,24,25]$. When seeking to extend this theory to higher dimensions, the natural next step is to consider maps defined over the quaternions; see e.g., [6, 7, 9, 15, 18, $20,22,26,30,31]$. The results obtained were, in a certain sense, slightly disappointing leading Bedding and Briggs [6] to make the following strong statement about the quadratic quaternionic map $\mathbf{f}(\mathrm{q})=\mathrm{q}^{2}+c$ : "We conclude that there is no new interesting dynamics in this map."

In this paper we deal with the extension of a quadratic map to the coquaternionic setting. More precisely, we consider the function of the coquaternioncic variable $\mathbf{f}(\mathbf{q})=q^{2}+c$, with $c$ the real parameter value for which the corresponding real map $f$ has a super-attractive 8 -cycle, and show that, even in this simple case, new and interesting features appear. In particular, we show that in addition to the real attractive cycle, four other coquaternionic attractive cycles coexist. Here, however, the cycles must be interpreted as cycles of sets of non-isolated points and an appropriate notion of attractivity has to be used.

We must refer that, since the algebra of coquaternions is isomorphic to the algebra $M_{2}(\mathbb{R})$ of $2 \times 2$ real matrices, the research on the iteration of functions defined on matrix algebras developed in [4] and [32] can also be seen - although with a different approach — as a first and important contribution to the study of the dynamics of coquaternionic maps.

The paper is organized as follows: in Section 2 we present a brief summary of the basic definitions and results on the algebra of coquaternions; Section 3 is dedicated to the study of the referred coquaternionic quadratic map and the main results of the paper are established; the concepts of attractive set cycles is here introduced and the existence of four coquaternionic attractive 8-set cycles, coexisting with the real attractive cycle, is shown; in Section 4, some investigation on the basins of attraction of the referred five 8-cycles is performed; finally, Section 5 concludes.

\section{Basic results}

In this section we introduce some notations and make a brief revision of the main results on coquaternions which we will use in the rest of the paper; for the proofs of the results and other details we refer the reader to $[1,21]$.

Let $\mathbb{R}$ denote the field of real numbers. The coquaternion algebra is an associative but noncommutative algebra over $\mathbb{R}$ defined as the set

$$
\mathbb{H}_{\mathrm{coq}}=\left\{q_{0}+q_{1} \mathrm{i}+q_{2} \mathrm{j}+q_{3} \mathrm{k}: q_{0}, q_{1}, q_{2}, q_{3} \in \mathbb{R}\right\},
$$

where $i, j, k$, called the imaginary units, satisfy

$$
\mathrm{i}^{2}=-1, \quad \mathrm{j}^{2}=\mathrm{k}^{2}=\mathrm{ijk}=1 .
$$

It follows easily that we have the following explicit expression for the product of two coquaternions $\mathrm{p}=$ $p_{0}+p_{1} \mathrm{i}+p_{2} \mathrm{j}+p_{3} \mathrm{k}$ and $\mathrm{q}=q_{0}+q_{1} \mathrm{i}+q_{2} \mathrm{j}+q_{3} \mathrm{k}$

$$
\begin{aligned}
\mathrm{pq}=p_{0} q_{0}-p_{1} q_{1}+p_{2} q_{2}+p_{3} q_{3}+\left(p_{0} q_{1}\right. & \left.+p_{1} q_{0}-p_{2} q_{3}+p_{3} q_{2}\right) \mathrm{i} \\
& +\left(p_{0} q_{2}-p_{1} q_{3}+p_{2} q_{0}+p_{3} q_{1}\right) \mathrm{j}+\left(p_{0} q_{3}+p_{1} q_{2}-p_{2} q_{1}+p_{3} q_{0}\right) \mathrm{k},
\end{aligned}
$$

from where we immediately obtain

$$
\mathbf{q}^{2}=q_{0}^{2}-q_{1}^{2}+q_{2}^{2}+q_{3}^{2}+2 q_{0}\left(q_{1} \mathbf{i}+q_{2} \mathbf{j}+q_{3} \mathbf{k}\right) .
$$

For a given coquaternion $q=q_{0}+q_{1} i+q_{2} j+q_{3} \mathrm{k} \in \mathbb{H}_{\text {coq }}$, we define, in analogy with the complex case, the real part of $\mathrm{q}$, Re $\mathrm{q}:=q_{0}$, the vector part of $\mathrm{q}$, Vec $\mathrm{q}:=q_{1} \mathrm{i}+q_{2} \mathrm{j}+q_{3} \mathrm{k}$ and the conjugate of $\mathrm{q}$, $\overline{\mathrm{q}}:=q_{0}-q_{1} \mathrm{i}-q_{2} \mathrm{j}-q_{3} \mathrm{k}$. We call determinant of $\mathrm{q}$, denoted by $\operatorname{det} \mathrm{q}$, to the quantity given by

$$
\operatorname{det} \mathrm{q}:=\mathrm{q} \overline{\mathrm{q}}=q_{0}^{2}+q_{1}^{2}-q_{2}^{2}-q_{3}^{2} .
$$

One can easily show that the function $\phi$ defined, for any $q=q_{0}+q_{1} \mathrm{i}+q_{2} \mathrm{j}+q_{3} \mathrm{k} \in \mathbb{H}_{\text {coq }}$, by

$$
\phi(\mathbf{q})=M_{\mathbf{q}}:=\left(\begin{array}{ll}
q_{0}+q_{3} & q_{1}+q_{2} \\
q_{2}-q_{1} & q_{0}-q_{3}
\end{array}\right)
$$


is an isomorphism between $\mathbb{H}_{\text {coq }}$ and the algebra $\mathcal{M}_{2}(\mathbb{R})$ of the $2 \times 2$ real matrices, the matrix $M_{\mathrm{q}}$ being called the matrix representation for the coquaternion $\mathrm{q}$. Some of the results for coquaternions can be established from known results for matrices, by invoking this isomorphism.

Remark 1. The choice of the term determinant for the quantity defined by (2) here adopted appears as very natural, by observing that this value is equal to the determinant of the matrix $M_{\mathrm{q}}{ }^{1}$

When convenient, we identify the coquaternion $\mathrm{q}=q_{0}+q_{1} \mathrm{i}+q_{2} \mathrm{j}+q_{3} \mathrm{k}$ with the element $\left(q_{0}, q_{1}, q_{2}, q_{3}\right)$ in $\mathbb{R}^{4}$. We will also consider three particularly important subspaces of dimension two of $\mathbb{H}_{\text {coq }}$, usually called the canonical planes or cycle planes. The first is $\left\{\mathrm{q} \in \mathbb{H}_{\mathrm{coq}}: \mathrm{q}=a+b \mathrm{i}, a, b \in \mathbb{R}\right\}$ which, naturally, we identify with the complex plane $\mathbb{C}$; the second, which we denote by $\mathbb{P}$ and whose elements are usually called perplex numbers is given by $\mathbb{P}=\left\{\mathrm{q} \in \mathbb{H}_{\text {coq }}: \mathrm{q}=a+b \mathrm{j}, a, b \in \mathbb{R}\right\}$ and corresponds to the classical Minkowski plane; the third, denoted by $\mathbb{D}$, is the subspace of the so-called dual numbers, $\mathbb{D}=\left\{\mathrm{q} \in \mathbb{H}_{\text {coq }}: \mathbf{q}=a+b(\mathrm{i}+\mathrm{j}), a, b \in \mathbb{R}\right\}$ and can be identified with the classical Laguerre plane.

Contrary to what happens with complex numbers and quaternions, not all nonzero coquaternions have an inverse; in fact, it can be shown that a coquaternion $q$ is invertible if and only if det $q \neq 0$, in which case $\mathrm{q}^{-1}=\frac{\overline{\mathrm{q}}}{\operatorname{det} \mathrm{q}}$

A concept which will play an important role in the dynamics of the map that we consider in this paper is now recalled: we say that a coquaternion $q$ is similar to a coquaternion $p$, and write $q \sim p$, if there exists an invertible coquaternion $h$ such that $p=h_{q h}{ }^{-1}$. This is an equivalence relation in $\mathbb{H}_{\text {coq }}$, partitioning $\mathbb{H}_{\text {coq }}$ in the so-called similarity classes. We denote by $[\mathrm{q}]$ the similarity class containing the coquaternion $\mathrm{q}$. It is easily shown (see e.g. [11]) that $[q]=\{q\}$ if and only if $q$ is a real number. The next result (see e.g. [11]) characterizes similar non-real coquaternions.

Theorem 1. Let $\mathrm{p}$ and $\mathrm{q}$ be two non-real coquaternions. Then $\mathrm{p} \sim \mathrm{q}$ if and only if

$$
\operatorname{Re} \mathrm{p}=\operatorname{Re} \mathrm{q} \quad \text { and } \operatorname{det}(\operatorname{Vec} \mathrm{p})=\operatorname{det}(\operatorname{Vec} \mathrm{q}) \text {. }
$$

In what follows, given a coquaternion $q$, we denote by $\mathrm{dv} q$ the determinant of the vector part of q, i.e. $\operatorname{dv} q:=\operatorname{det}(\operatorname{Vec} p)$.

Theorem 1 shows us that, for any non-real coquaternion $q=q_{0}+V e c q$, we have:

$$
[\mathbf{q}]=\left\{x \in \mathbb{H}_{\mathrm{coq}}: x_{0}=q_{0} \text { and } x_{1}^{2}-x^{2}-x_{3}^{2}=\mathrm{dv} \mathbf{q}\right\} \backslash\left\{q_{0}\right\} .
$$

We can thus identify the similarity class of a non-real coquaternion q with an hyperboloid in the hyperplane $\left\{\left(x_{0}, x_{1}, x_{2}, x_{3}\right) \in \mathbb{R}^{4}: x_{0}=q_{0}\right\}$ : this will be an hyperboloid of two-sheets if $\mathrm{dv} \mathrm{q}>0$, an hyperboloid of one-sheet, if $\mathrm{dvq}<0$ or a degenerate hyperboloid (cone), if $\mathrm{dv} \mathrm{q}=0$. $^{2}$

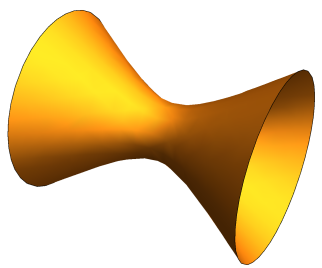

$(a)$

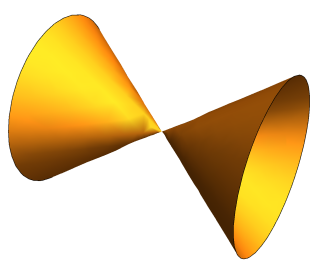

$(b)$

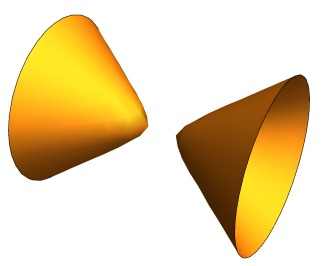

$(c)$

Figure 1: Plots, in the hyperplane $\left\{\left(x_{0}, x_{1}, x_{2}, x_{3}\right) \in \mathbb{R}^{4}: x_{0}=q_{0}\right\}$, of the similarity class of a coquaternion q. $(a) \mathrm{dv} \mathrm{q}<0 ;(b) \mathrm{dv} \mathrm{q}=0 ;(c) \mathrm{dv} \mathrm{q}>0$.

The next result is an immediate consequence of Theorem 1.

\footnotetext{
${ }^{1}$ We must refer that other authors use different denominations and notations.

${ }^{2}$ Strictly speaking, in the case $d v q=0$, the vertex of the cone does not belong to $[q]$.
} 
Corollary 1. Let $\mathrm{q}=q_{0}+$ Vec $\mathrm{q}$ be a non-real coquaternion. Then $[\mathrm{q}]=\left[\mathrm{q}_{\mathrm{s}}\right]$ where

$$
\mathrm{q}_{\mathrm{s}}= \begin{cases}q_{0}+\sqrt{\mathrm{dvq} i}, & \text { if } \mathrm{dv} \mathrm{q}>0, \\ q_{0}+\sqrt{-d v q} \mathrm{j}, & \text { if } \mathrm{dv} \mathrm{q}<0, \\ q_{0}+\mathrm{i}+\mathrm{j}, & \text { if } \mathrm{dv} \mathrm{q}=0 .\end{cases}
$$

The coquaternion $\mathrm{q}_{\mathrm{S}}$ given by (4) will be referred to as the standard representative of the similarity class of $\mathrm{q}$. Note that the representative is a complex number, a perplex number or a dual number, according to $d v q>0, d v q<0$ or $d v q=0$, respectively.

\section{The dynamics of a coquaternionic quadratic map}

All our results are concerned with the dynamics of a quadratic coquaternionic map of the form

$$
\begin{aligned}
\mathbf{f}: \mathbb{H}_{\mathrm{coq}} \rightarrow \mathbb{H}_{\mathrm{coq}} \\
\quad \mathrm{q} \mapsto \mathrm{q}^{2}+c,
\end{aligned}
$$

with $c$ a real parameter to be fixed later. We first recall some basic definitions, essentially to fix our notation. For $k \in \mathbb{N}$, we shall denote by $\mathbf{f}^{k}$ the $k$-th iterate of $\mathbf{f}$, inductively defined by $\mathbf{f}^{0}=\operatorname{id}_{\mathbb{H}_{\text {coq }}}$ and $\mathbf{f}^{k}=\mathbf{f} \circ \mathbf{f}^{k-1}$. For a given initial point $\mathbf{q}_{0} \in \mathbb{H}_{\text {coq }}$, the orbit of $\mathbf{q}_{0}$ under $\mathbf{f}$ is the sequence $\mathcal{O}\left(\mathbf{q}_{0}\right):=\left(\mathbf{f}^{k}\left(\mathbf{q}_{0}\right)\right)_{k \in \mathbb{N}_{0}}$. A point $\mathrm{q} \in \mathbb{H}_{\text {coq }}$ is said to be a periodic point of $\mathbf{f}$ with period $n \in \mathbb{N}$, if $\mathbf{f}^{n}(\mathbf{q})=\mathbf{q}$, with $\mathbf{f}^{k}(\mathbf{q}) \neq \mathbf{q}$ for $0<k<n$; in this case, we say that the set $\left\{\mathbf{q}, \mathbf{f}(\mathbf{q}), \ldots, \mathbf{f}^{n-1}(\mathbf{q})\right\}$ is a $n$-cycle for $\mathbf{f}$, usually written as $\mathrm{q}_{0} \stackrel{\mathbf{f}}{\rightarrow} \mathrm{q}_{1} \stackrel{\mathbf{f}}{\rightarrow} \cdots \stackrel{\mathbf{f}}{\rightarrow} \mathrm{q}_{n-1}$ with $\mathrm{q}_{i}=\mathbf{f}^{i}(\mathbf{q})$. Periodic points of period one are called fixed points.

Let $\mathrm{q}^{*}$ be a fixed point of $\mathbf{f}$. Then the basin of attraction $\mathcal{B}\left(\mathrm{q}^{*}\right)$ of $\mathrm{q}^{*}$ is defined as

$$
\mathcal{B}\left(\mathbf{q}^{*}\right):=\left\{\mathbf{q}: \lim _{k \rightarrow \infty} \mathbf{f}^{k}(\mathbf{q})=\mathbf{q}^{*}\right\} .
$$

Here, the convergence of the sequence $\left(\mathbf{f}^{k}(\mathbf{q})\right)_{k}$ is to be understood with respect to any norm in $\mathbb{R}^{4}$, for example the Euclidean norm. $\mathbf{f}^{n}$.

The basin of attraction of a $n$-periodic point $\mathrm{q}^{*}$ is defined as the basin of attraction of $\mathrm{q}^{*}$ under the map

The basin of attraction of a $n$-cycle $\mathscr{C}=\left\{\mathrm{q}_{0}, \mathrm{q}_{1}, \ldots, \mathrm{q}_{n-1}\right\}$ is given by

$$
\mathcal{B}(\mathscr{C})=\bigcup_{i=0}^{n-1} \mathcal{B}\left(\mathbf{q}_{i}\right)
$$

We first observe that, since $c \in \mathbb{R}$, we have, for any invertible $\mathrm{h} \in \mathbb{H}_{\text {coq }}$,

$$
\mathbf{f}\left(\mathrm{h}^{-1} \mathrm{qh}\right)=\mathrm{h}^{-1} \mathrm{q}^{2} \mathrm{~h}+c=\mathrm{h}^{-1} \mathrm{q}^{2} \mathrm{~h}+\mathrm{h}^{-1} c h=\mathrm{h}^{-1} \mathbf{f}(\mathrm{q}) \mathrm{h},
$$

from where we immediately obtain

$$
\mathbf{f}^{n}\left(\mathrm{~h}^{-1} \mathrm{qh}\right)=\mathrm{h}^{-1} \mathbf{f}^{n}(\mathbf{q}) \mathrm{h} .
$$

This shows that if a coquaternion $\mathrm{q}$ is a periodic point of period $n$ of the map $\mathbf{f}$, the same is true for all the coquaternions similar to $\mathrm{q}$. In other words, if $\mathrm{q}$ is a periodic point of period $n$ of the map $\mathbf{f}$, then the whole similarity class [q] (which, recall, constitutes an hyperboloid, whenever $q \notin \mathbb{R}$ ) is made up of periodic points of period $n$ of $\mathbf{f}$. Hence, for all non-real periodic points of the map $\mathbf{f}$, we now have a situation not present in the classical case of the real/complex quadratic maps: they constitute sets of non-isolated points. So, in this case, it makes more sense to talk about $n$-cycles of sets, instead of $n$-cycles of isolated points $\left\{\mathbf{q}_{0}, \ldots, \mathrm{q}_{n-1}\right\}$.

Definition 1. We say that the sets $\mathcal{S}_{0}, \ldots, \mathcal{S}_{n-1}$ form a $n$-set cycle for the map $\mathbf{f}$ and write $\mathcal{S}_{0} \stackrel{\mathbf{f}}{\rightarrow} \mathcal{S}_{1} \stackrel{\mathbf{f}}{\rightarrow}$ $\ldots \stackrel{\mathbf{f}}{\rightarrow} \mathcal{S}_{n-1}$, if each of the sets $\mathcal{S}_{i}$ is formed by periodic points of period $n$ of $\mathbf{f}$ and, for any $\mathrm{q} \in \mathcal{S}_{i}$, the corresponding image, $\mathbf{f}(\mathrm{q})$, belongs to the set $\mathcal{S}_{i+1}$, with $\mathcal{S}_{n}:=\mathcal{S}_{0}$. 
Remark 2. When the map $\mathbf{f}$ is implicit from the context, we will simply write $\mathcal{S}_{0} \rightarrow \mathcal{S}_{1} \rightarrow \cdots \rightarrow \mathcal{S}_{n-1}$.

Since non-real periodic points of the map $\mathbf{f}$, when existing, form sets of non-isolated points, it is simple to recognize that they cannot be attractive in the usual sense. Consider, for example, the case of a set of non-isolated fixed points: given a point $\mathrm{q}^{*}$ in this set, any neighborhood of $\mathrm{q}^{*}$ always contains other fixed points, and so it is impossible to find a neighborhood of $\mathrm{q}^{*}$ totally formed by points whose dynamics evolve to $q^{*}$. Hence, it becomes necessary to introduce a new notion of attractivity; see e.g. [3]. We propose to adopt the following definition.

Definition 2. A set $\mathcal{S}$ of non-isolated periodic points of period $n \in \mathbb{N}$ of the coquaternionic map $\mathbf{f}$ is said to be an attractive set if, given any coquaternion q sufficiently close to the set $\mathcal{S}$, the sequence of iterates $\left(\left(\mathbf{f}^{n}\right)^{k}(\mathbf{q})\right)_{k}$ converges to a point belonging to $\mathcal{S}$.

If a $n$-set cycle $\mathcal{S}_{0} \rightarrow \mathcal{S}_{1} \rightarrow \cdots \rightarrow \mathcal{S}_{n-1}$ for $\mathbf{f}$ is such that each of the sets $\mathcal{S}_{i}$ is attractive, then we will say that the sets $\mathcal{S}_{0}, \ldots, \mathcal{S}_{n-1}$ form an attractive $n$-set cycle.

In what follows, we will denote by $f_{\mathbb{C}}$ the complex map obtained by restricting the map $f$ to the complex plane i.e. $f_{\mathbb{C}}:=\mathbf{f}_{\mid \mathbb{C}}$; the restriction of the map $\mathbf{f}$ to the reals will simply be denoted by $f$, i.e. $f:=\mathbf{f}_{\mid \mathbb{R}}$. Note that $f_{\mathbb{C}}$ is a complex-valued map and $f$ is real-valued map.

\section{The attractive cycles of the quadratic map $f$}

The specific value of the parameter $c$ we are going to consider is a value for which the corresponding real map $f$ has a super-attractive 8 -cycle: ${ }^{3}$

$$
c=-1.3815474844320625 \ldots
$$

We claim that, apart from the real 8-cycle, which we will denote by $\mathscr{C}$, the map $\mathbf{f}$ has also four attractive 8-set cycles, namely

$$
\begin{array}{cl}
\mathscr{C}_{1}: & \mathcal{H}_{1,0} \rightarrow \mathcal{H}_{1,1} \rightarrow \mathcal{H}_{1,2} \rightarrow \mathcal{H}_{1,3} \rightarrow \mathcal{H}_{1,4} \rightarrow \mathcal{H}_{1,5} \rightarrow \mathcal{H}_{1,6} \rightarrow \mathcal{H}_{1,7} \\
\mathscr{C}_{2}: & \mathcal{H}_{2,0} \rightarrow \mathcal{H}_{2,1} \rightarrow \mathcal{H}_{2,2} \rightarrow \mathcal{H}_{2,3} \rightarrow \mathcal{H}_{2,4} \rightarrow \mathcal{H}_{2,5} \rightarrow \mathcal{H}_{2,6} \rightarrow \mathcal{H}_{2,7} \\
\mathscr{C}_{3}: & \mathcal{H}_{3,0} \rightarrow \mathcal{H}_{3,1} \rightarrow \mathcal{H}_{3,2} \rightarrow \mathcal{H}_{3,3} \rightarrow \mathcal{H}_{3,4} \rightarrow \mathcal{H}_{3,5} \rightarrow \mathcal{H}_{3,6} \rightarrow \mathcal{H}_{3,7} \\
\mathscr{C}_{4}: & \mathcal{H}_{4,0} \rightarrow \mathcal{H}_{4,1} \rightarrow \mathcal{H}_{4,2} \rightarrow \mathcal{H}_{4,3} \rightarrow \mathcal{H}_{4,0} \rightarrow \mathcal{H}_{4,1} \rightarrow \mathcal{H}_{4,2} \rightarrow \mathcal{H}_{4,3}
\end{array}
$$

where $\mathcal{H}_{i, j}$ are the similarity classes given in Table $1 .{ }^{4}$

That the sets given in Table 1 form 8-set cycles was not difficult to verify: in each case, we just had to determine the evolution of the standard representative $\mathrm{q}_{i 0}$ of the first class $\mathcal{H}_{i 0}$ and confirm that the successive iterates belonged to the similarity classes indicated as forming the cycle and also that $\mathbf{f}^{8}\left(\mathrm{q}_{i 0}\right)=\mathrm{q}_{i 0}$. Note that this implies that $\mathbf{f}^{8}\left(\mathbf{f}^{k}\left(\mathbf{q}_{i 0}\right)\right)=\mathbf{f}^{k}\left(\mathbf{q}_{i 0}\right)$ and, since all points of the classes share the same dynamics, one can conclude that all the classes are made up of periodic points of period 8 .

To verify if the referred coquaternionic cycles were attractive, we used the following computational procedure: a coquaternionic perturbation with each component with a modulus less than 0.001 randomly chosen was added to the representative of each class and 80 iterations, starting with this perturbed seed, were performed; then, the last iteration was analyzed and it was checked whether or not it belonged to the similarity class of the unperturbed representative; this process was repeated 1000000 times. Since the answer was always positive, although we do not have a formal analytic proof of the fact that the cycles are attractive, we can state, with great confidence, that this is true.

Remark 3. We emphasize that the representatives of the sets of each set cycle do not necessarily form a 8 -cycle of points. This is what happens, in particular, with the cycle $\mathscr{C}_{4}$ where the sets $\mathcal{H}_{4,0}, \mathcal{H}_{4,1}, \mathcal{H}_{4,2}$ and $\mathcal{H}_{4,3}$ appear repeated, but do not form a 4-cycle; any element $\mathrm{q} \in \mathcal{H}_{4,0}$ is such that its fourth iterate belongs to the same set $\mathcal{H}_{4,0}$; however, we do not have $\mathbf{f}^{4}(\mathbf{q})=\mathbf{q}$, but $\mathbf{f}^{4}(\mathbf{q})=\overline{\mathbf{q}}$, instead; only after 8 iterations the element $\mathrm{q}$ repeats itself.

\footnotetext{
${ }^{3}$ Recall that a $n$-cycle $x_{0} \rightarrow x_{1} \rightarrow \cdots \rightarrow x_{n-1}$ for the real map $f(x)=x^{2}+c$ is called super-attractive if $\left|\left(f^{n}\right)^{\prime}\left(x_{k}\right)\right|=$ $\prod_{i=0}^{n-1}\left|f^{\prime}\left(x_{i}\right)\right|=0$, for $k=0,1, \ldots, n-1$; in that case, the cycle contains the critical point $x=0$ of $f$.

${ }^{4}$ The representatives of the classes, given in Table 1 with 12 digits, were computed using an approximation to the value of $c$ with 16 digits of accuracy.
} 


\begin{tabular}{|l|l|}
\hline Cycle & Sets \\
\hline $\mathscr{C}_{1}$ & $\mathcal{H}_{1,0}=[-0.690773742216+0.690773742216 \mathrm{j}]$ \\
& $\mathcal{H}_{1,1}=[-0.427210758562+0.954336725870 \mathrm{j}]$ \\
& $\mathcal{H}_{1,2}=[-0.288279865856+0.815405833165 \mathrm{j}]$ \\
& $\mathcal{H}_{1,3}=[-0.633555530615+0.470130168406 \mathrm{j}]$ \\
& $\mathcal{H}_{1,4}=[-0.759132498814+0.595707136605 \mathrm{j}]$ \\
& $\mathcal{H}_{1,5}=[-0.450398341074+0.904441294345 \mathrm{j}]$ \\
& $\mathcal{H}_{1,6}=[-0.360674763873+0.814717717144 \mathrm{j}]$ \\
& $\mathcal{H}_{1,7}=[-0.587696240509+0.587696240509 \mathrm{j}]$ \\
\hline $\mathscr{C}_{2}$ & $\mathcal{H}_{2,0}=[0.263562983654+0.263562983654 \mathrm{j}]$ \\
& $\mathcal{H}_{2,1}=[-1.24261659173+0.138930892705 \mathrm{j}]$ \\
& $\mathcal{H}_{2,2}=[0.181850302550+0.345275664758 \mathrm{j}]$ \\
& $\mathcal{H}_{2,3}=[-1.22926266722+0.125576968199 \mathrm{j}]$ \\
& $\mathcal{H}_{2,4}=[0.145308795531+0.308734157739 \mathrm{j}]$ \\
& $\mathcal{H}_{2,5}=[-1.26511605822+0.0897235772009 \mathrm{j}]$ \\
& $\mathcal{H}_{2,6}=[0.227021476635+0.227021476635 \mathrm{j}]$ \\
& $\mathcal{H}_{2,7}=[-1.27846998272+0.103077501707 \mathrm{j}]$ \\
\hline $\mathscr{C}_{3}$ & $\mathcal{H}_{3,0}=[-0.677419817710+0.677419817710 \mathrm{j}]$ \\
& $\mathcal{H}_{3,1}=[-0.463752265581+0.917795218851 \mathrm{j}]$ \\
& $\mathcal{H}_{3,2}=[-0.324133256854+0.851259224163 \mathrm{j}]$ \\
& $\mathcal{H}_{3,3}=[-0.551842849511+0.551842849511 \mathrm{j}]$ \\
& $\mathcal{H}_{3,4}=[-0.772486423320+0.609061061112 \mathrm{j}]$ \\
& $\mathcal{H}_{3,5}=[-0.413856834055+0.940982801364 \mathrm{j}]$ \\
& $\mathcal{H}_{3,6}=[-0.324821372875+0.778864326146 \mathrm{j}]$ \\
& $\mathcal{H}_{3,7}=[-0.669408921613+0.505983559405 \mathrm{j}]$ \\
\hline $\mathscr{C}_{4}$ & $\mathcal{H}_{4,0}=[-0.0817126811041+0.0817126811041 \mathrm{j}]$ \\
& $\mathcal{H}_{4,1}=[-1.36819355993-0.0133539245065 \mathrm{j}]$ \\
& $\mathcal{H}_{4,2}=[0.490584460290+0.0365415070189 \mathrm{j}]$ \\
& $\mathcal{H}_{4,3}=[-1.13953909002+0.0358533909981 \mathrm{j}]$ \\
\hline
\end{tabular}

Table 1: Coquaternionic 8-set cycles

Finally, based on a large number of computations with randomly chosen initial seeds in $\mathbb{H}_{\text {coq }}$, we have reasons to believe that the only attractive cycles of the map $f$ are the five 8-cycles referred previously, i.e. that the map $f$ has no other attractive cycle of any period.

\section{Basins of attraction of the 8-cycles}

The results of the previous section have shown a completely different behavior of the coquaternionic quadratic map $\mathbf{f}$ when compared with the corresponding complex map $f_{\mathbb{C}}$ : the existence of more than one attractive cycle. The purpose of this section is to make some investigation on the basins of attraction of the five 8-cycles of the map $\mathbf{f}$ previously identified.

It is well-known that the basin of attraction of the real cycle $\mathscr{C}$ for the real map $f$ or even for the complex quadratic map $f_{\mathbb{C}}$ is a bounded set; see e.g. [29]. We now show that this is not the case when we consider the extension of this map to the set of coquaternions, by choosing a particular type of coquaternions as seeds. In what follows, we will denote by $\mathcal{B}_{\mathbb{R}}(\mathscr{C})$ the real basin of attraction of the real 8-cycle $\mathscr{C}$ i.e. $\mathcal{B}_{\mathbb{R}}(\mathscr{C})=\mathcal{B}(\mathscr{C}) \cap \mathbb{R}$.

Let $\mathrm{q}$ be a coquaternion dual number, $\mathrm{q}=x_{0}+\alpha(\mathrm{i}+\mathrm{j})$. From (1), one can deduce the following expression for the $n$th iteration of $\mathrm{q}$ :

$$
\begin{aligned}
\mathbf{f}^{n}(\mathbf{q}) & =f^{n}\left(x_{0}\right)+\left(\prod_{k=0}^{n-1} f^{\prime}\left(x_{k}\right)\right) \alpha(\mathbf{i}+\mathbf{j}), \quad x_{k}=f^{k}\left(x_{0}\right), \\
& =f^{n}\left(x_{0}\right)+\left(f^{n}\right)^{\prime}\left(x_{0}\right) \alpha(\mathbf{i}+\mathbf{j}) .
\end{aligned}
$$


Since the parameter $c$ is a regular parameter ${ }^{5}$ of the real map $f$, then, for any $x_{0}$ in the real basin of attraction of this cycle, we have $\lim _{n \rightarrow \infty}\left(f^{n}\right)^{\prime}\left(x_{0}\right)=0$; see e.g. [12]. This means that a coquaternion of the form $\mathrm{q}=x_{0}+\alpha \mathrm{i}+\alpha \mathrm{j}$, with $x_{0} \in \mathcal{B}_{\mathbb{R}}(\mathscr{C})$ and $\alpha$ an arbitrarily chosen real number, belongs to $\mathcal{B}(\mathscr{C})$, showing that this basin is not bounded.

We now present some plots of the basins of attraction of the five attractive 8-cycles of the quadratic map f. Since the basins are 4-dimensional sets, they require to be submersed from $\mathbb{R}^{4}$ to $\mathbb{R}^{3}$ or to $\mathbb{R}^{2}$ for drawing purposes. The representations given in our figures are two-dimensional plots obtained by always assuming that $q_{3}=0$, and then considering different two-dimensional slices of the three-dimensional sets thus obtained; the slices are created by either fixing different values for $q_{2}$ or by fixing different values for $q_{1}$, i.e. all the figures correspond to plots in planes which are either parallel to the complex plane or parallel to the Minkowski plane. Note that all the plots obtained in this way are symmetric with respect to the real axis and with respect to the imaginary axis (respectively j-axis) of the plane $q_{3}=0$ and $q_{2}=\alpha$ (respectively $q_{3}=0$ and $q_{1}=\beta$ ) where the plot is considered. In fact, from (1) and (2), one easily deduce that if $\mathbf{q}=q_{0}+q_{1} \mathrm{i}+q_{2} \mathrm{j}$ and $\mathbf{q}^{*}=-q_{0}+q_{1} \mathbf{i}+q_{2} \mathbf{j}$, then $\mathbf{f}\left(\mathbf{q}^{*}\right)=\mathbf{f}(\overline{\mathbf{q}})$; since $\mathbf{f}(\overline{\mathbf{q}}) \sim \mathbf{f}(\mathbf{q})$, we conclude that $\mathrm{q}^{*}$ and $\mathrm{q}$ have the same dynamics. The symmetry with respect to the real axis follows immediately by observing that $q=q_{0}+q_{1} \mathrm{i}+q_{2} \mathrm{j}$ and $\mathrm{q}^{*}=q_{0}-q_{1} \mathrm{i}+q_{2} \mathrm{j}$ (respectively $\mathrm{q}^{*}=q_{0}+q_{1} \mathrm{i}-q_{2} \mathrm{j}$ ) are similar coquaternions.

In all the plots, the points in the basin of attraction of the real cycle $\mathscr{C}$ are colored in red and the colors cyan, yellow, gray and pink are used for the basins of attraction of the cycles $\mathscr{C}_{1}, \mathscr{C}_{2}, \mathscr{C}_{3}$ and $\mathscr{C}_{4}$, respectively; the points corresponding to diverging dynamics are represented in black.

Figures 2 - ?? show plots in planes parallel to the complex plane; more specifically, Fig. 2 contains the plots obtained with $q_{2}=0,0.1,0.2$, Fig. 3 corresponds to $q_{2}=0.4,0.6,0.8$ and in Fig. 4 we used $q_{2}=1.0,1.2,1.6,1.9$.

For the first choice, $q_{2}=0$, we immediately recognize the picture associated with the dynamics of the quadratic complex map. ${ }^{6}$ As we increase the value of $q_{2}$, the four coquaternionic basins of attraction emerge, showing a remarkable intertwined structure.

We now point out and explain some of the features we observe in this first set of figures (Fig. 2 to Fig. 4), having in mind that each of the plots corresponds to $q_{3}=0$ and a fixed value $\alpha$ of $q_{2}$.

We first recall a known result for the real map $f(x)=x^{2}+c$ with our choice of parameter $c$ : the interval $I=(-a, a)$, with $a$ the positive root of $x^{2}-x+c=0$, i.e. $a=1.7773204313844129 \ldots$, coincides, with the exception of a set of isolated points, with the real basin of attraction of the attractive 8-cycle $\mathscr{C}, \mathcal{B}_{\mathbb{R}}(\mathscr{C})$. Also, as previously shown, any coquaternion of the form $q_{0}+\alpha \mathrm{i}+\alpha \mathrm{j}$ with $q_{0}$ belonging to $\mathcal{B}_{\mathbb{R}}(\mathscr{C})$ will converge to the real cycle $\mathscr{C}$. These two facts explain the appearance in all the plots of a red horizontal line $q_{1}=q_{2}=\alpha$ stretching from $q_{0}=-a$ to $q_{0}=a .^{7}$ Naturally, due to the symmetry with respect to the real axis, a line for $q_{1}=-\alpha$ also appears. As $\alpha$ increases, these two lines become more and more apart.

From the expression (1) it is simple to verify that, for any coquaternion $q$ of the form $q=q_{1} i+\alpha j$ (i.e. a point in the imaginary axis of the plot), we have $\mathbf{f}(\mathbf{q})=\alpha^{2}-q_{1}^{2}+c$, showing that the first iterate of such a $\mathrm{q}$ is a real number. This shows that the dynamics of $\mathrm{q}$ is the dynamics of the point $x_{0}=\alpha^{2}-q_{1}^{2}+c$ with respect to the real map $f$. Hence, $\mathrm{q}=q_{1} \mathrm{i}+\alpha \mathrm{j}$ will belong to the basin of attraction of the real cycle, $\mathcal{B}(\mathscr{C})$, if and only if $\alpha^{2}-q_{1}^{2}+c \in \mathcal{B}_{\mathbb{R}}(\mathscr{C})$. Therefore, with the exception of a set of isolated points (not distinguishable in the plots), all the points of the form $\mathrm{q}=q_{1} \mathrm{i}+\alpha \mathrm{j}$ with $q_{1}$ satisfying $-a<\alpha^{2}-q_{1}^{2}+c<a$, or equivalently

$$
\alpha^{2}+c-a<q_{1}^{2}<\alpha^{2}+c+a,
$$

will be painted in red. Since $c+a$ is a positive quantity, the second inequality is satisfied for $q_{1}$ belonging to the interval $\left(-\sqrt{\alpha^{2}+c+a}, \sqrt{\alpha^{2}+c+a}\right)$. Now, for $|\alpha|<a$, we have $\alpha^{2}+c-a<a^{2}+c-a=0$, which means that the first inequality is trivially satisfied; when $|\alpha|>a$, the first inequality imposes an additional restriction on the points in the imaginary axis which will appear in red: $\left|q_{1}\right|>\sqrt{\alpha^{2}+c-a}$. This is very apparent in the plots in Fig 4: in the first three plots, which correspond to values of $\alpha$ satisfying $|\alpha|<a$, there is no interruption in the red vertical line (the line stretches from $q_{1}=-\sqrt{\alpha^{2}+c+a}$ to $q_{1}=\sqrt{\alpha^{2}+c+a}$ );

\footnotetext{
${ }^{5}$ The parameter $c$ for the real map $f_{c}(x)=x^{2}+c$ is said to be regular if the map $f_{c}$ has a $n$-cycle $\alpha_{0} \rightarrow \alpha_{1} \rightarrow \cdots \rightarrow \alpha_{n-1}$ for which $\left|\left(f^{n}\right)^{\prime}\left(\alpha_{0}\right)\right|<1$; see [13].

${ }^{6}$ We observe that some isolated points which neither belong to $\mathcal{B}(\mathscr{C})$ nor have a diverging dynamics and which, as such, should be uncolored, due to the grid we use to construct the basins are not distinguishable; in other words, the red set in the plot corresponds to the representation of filled-in Julia set for $f$.

${ }^{7}$ Here again, some isolated points, which have a divergent dynamics, do not appear in the "continuous" red line, due to the regularity of the grid used to compute the basins.
} 

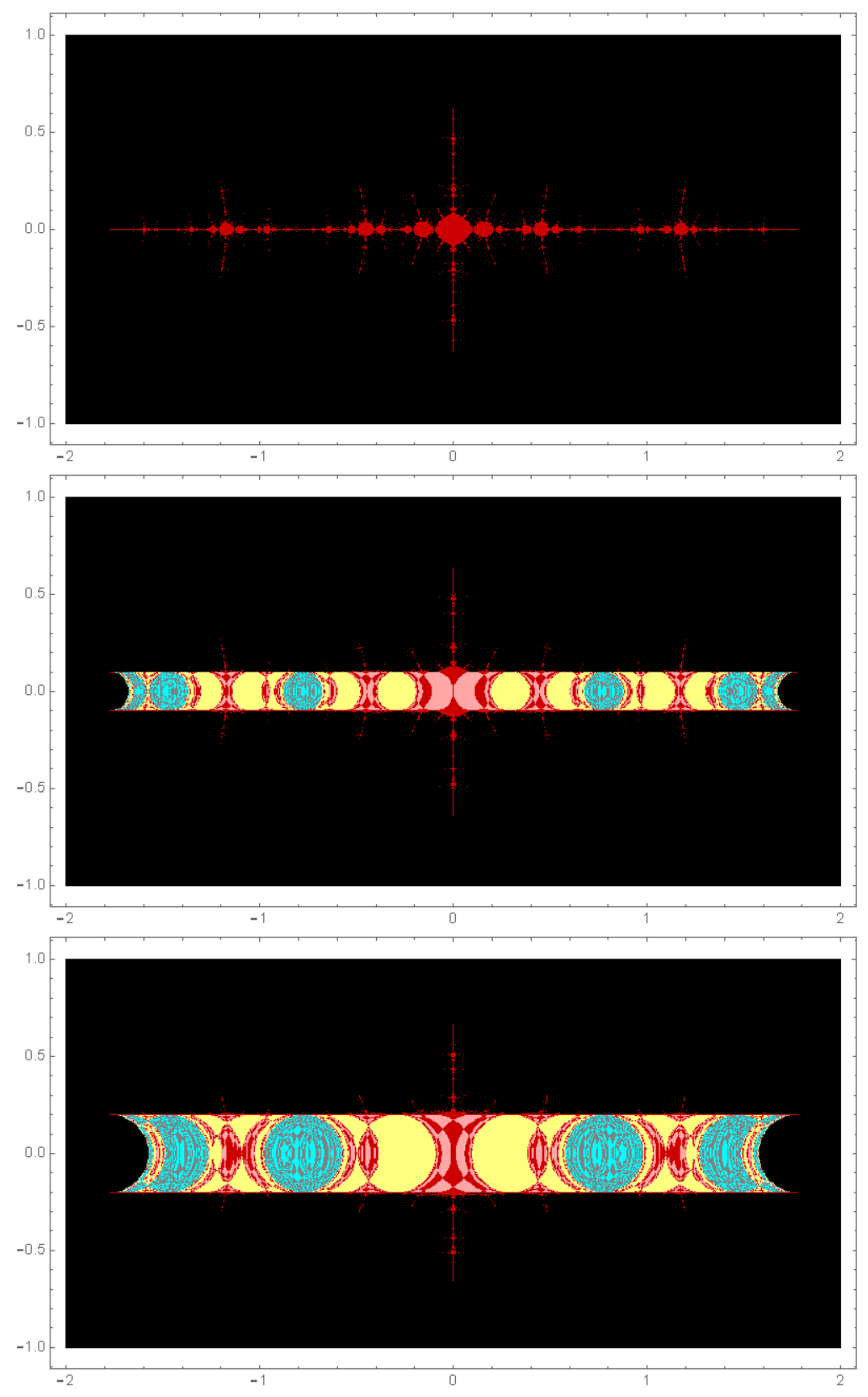

Figure 2: Plots, in different planes parallel to the complex plane, of the basins of attraction of the real cycle $\mathscr{C}$ (red) and of the coquaternionic cycles $\mathscr{C}_{1}$ (cyan), $\mathscr{C}_{2}$ (yellow), $\mathscr{C}_{3}$ (gray) and $\mathscr{C}_{4}$ (pink). Top: $q_{2}=0$ (complex plane); middle: $q_{2}=0.1$; bottom: $q_{2}=0.2$.

however, in the last plot corresponding to $\alpha=1.9>a$, the red vertical line is split in two pieces: from $q_{1}=-\sqrt{\alpha^{2}+c-a}$ to $q_{1}=-\sqrt{\alpha^{2}+c+a}$ and from to $q_{1}=\sqrt{\alpha^{2}+c-a}$ to $q_{1}=\sqrt{\alpha^{2}+c+a}$.

In our last figure, Figure ??, we show plots obtained in planes parallel to the Minkowsi plane; more precisely, the plots were obtained considering $q_{3}=0$ and $q_{1}=\beta$, for $\beta=0,0.1,0.6,0.7$. An analysis similar to the one carried out for the plots in planes parallel to the complex plane explains easily the appearance of the horizontal lines $q_{1}=q_{2}=\beta$ in each of these new plots. Also, in this case, it is easy to show that, with the exception of isolated points, if $|\beta|<\sqrt{a+c}=0.6291048775461452 \ldots$, the points of the form $\beta$ i $+q_{2}$ j (i.e. points in the $\mathrm{j}$-axis in the plots) with $q_{2}$ belonging to the interval $\left(-\sqrt{\beta^{2}+a-c}, \sqrt{\beta^{2}+a-c}\right)$ will appear in red, whilst, if $|\beta|>\sqrt{a+c}$, the same will happen to points with $q_{2}$ lying in the union of the two intervals 

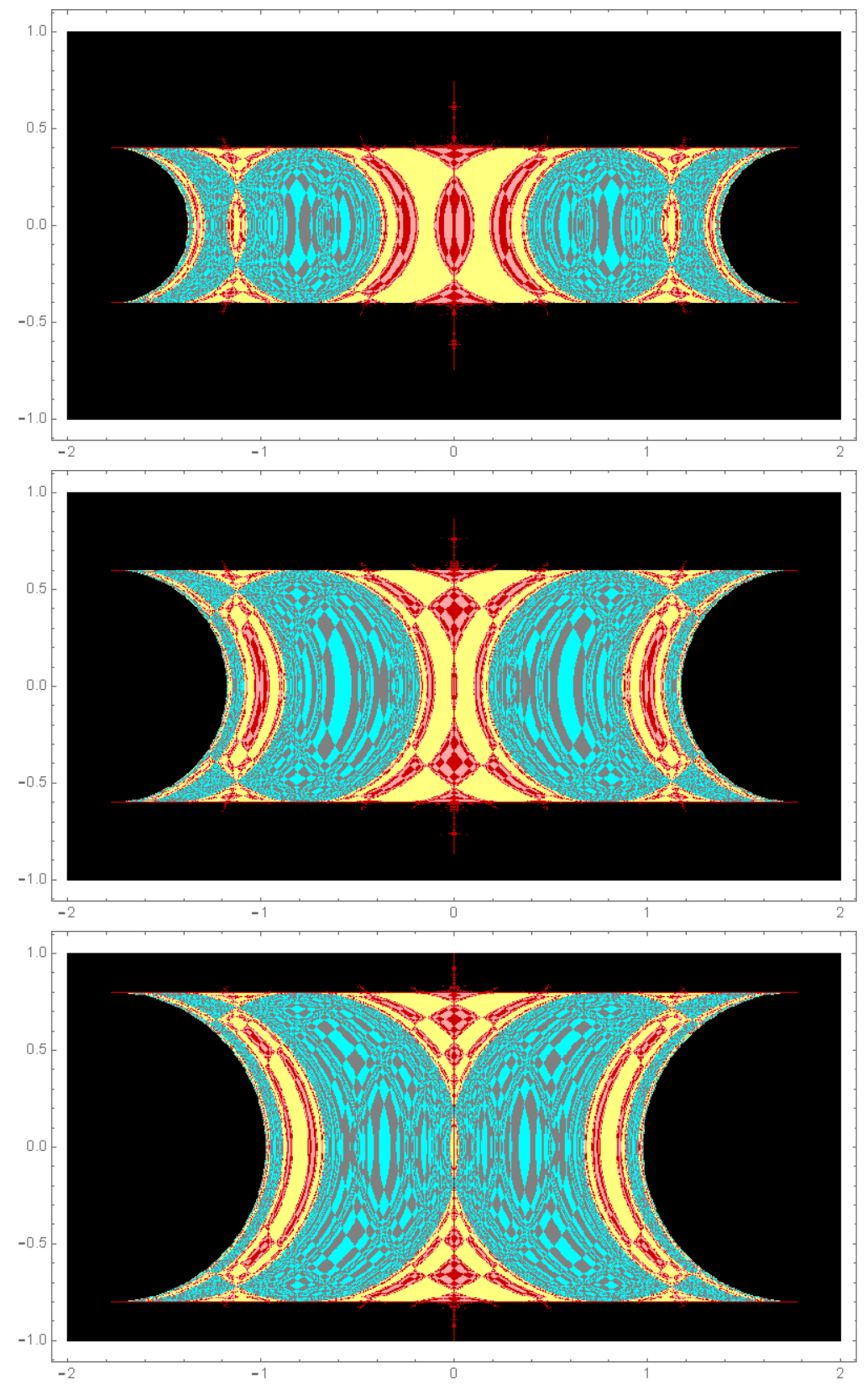

Figure 3: Plots, in different planes parallel to the complex plane, of the basins of attraction of the real cycle $\mathscr{C}$ (red) and of the coquaternionic cycles $\mathscr{C}_{1}$ (cyan), $\mathscr{C}_{2}$ (yellow), $\mathscr{C}_{3}$ (gray) and $\mathscr{C}_{4}$ (pink). Top: $q_{2}=0.4$; middle: $q_{2}=0.6$; bottom: $q_{2}=0.8$.

$\left(-\sqrt{\beta^{2}+a-c},-\sqrt{\beta^{2}-a-c}\right)$ and $\left(\sqrt{\beta^{2}-a-c}, \sqrt{\beta^{2}+a-c}\right)$. This is very clearly shown in the figure: in the first three plots, corresponding to values of $\beta$ such that $|\beta|<\sqrt{a+c}$, there is no interruption of the red vertical line, which stretches from $q_{2}=-\sqrt{\beta^{2}+a-c}$ to $q_{2}=\sqrt{\beta^{2}+a-c}$, but this is no longer true in the last plot, corresponding to $\beta=0.7>\sqrt{a+c}$. 

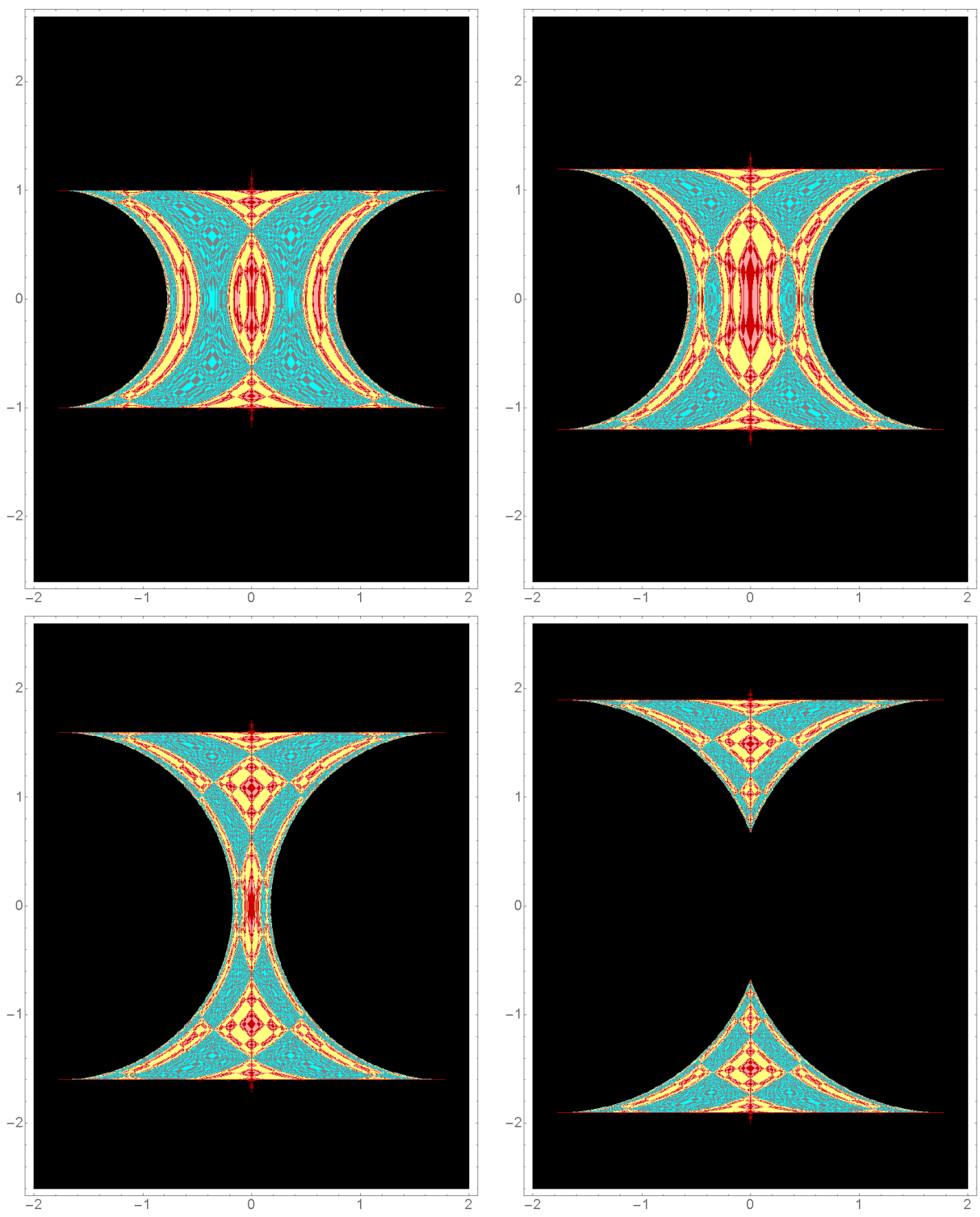

Figure 4: Plots, in different planes parallel to the complex plane, of the basins of attraction of the real cycle $\mathscr{C}$ (red) and of the coquaternionic cycles $\mathscr{C}_{1}$ (cyan), $\mathscr{C}_{2}$ (yellow), $\mathscr{C}_{3}$ (gray) and $\mathscr{C}_{4}$ (pink). Top-left: $q_{2}=1.0$; top-right: $q_{2}=1.2$; bottom-left: $q_{2}=1.6$; bottom-right: $q_{2}=1.9$.

\section{Conclusions}

In this paper we considered the coquaternionic quadratic map $\mathbf{f}(\mathbf{q})=\mathbf{q}^{2}+c$, with $c$ the parameter value for which the corresponding real map has a real super-attractive 8-cycle. We have shown that, in addition to the real cycle, this coquaternionic map has sets of non-isolated periodic points of period 8 which form four attractive 8-cycles of sets (with a notion of attractivity appropriately adapted to deal with non-isolated sets of points). Some characteristics of the basins of attraction of the attracting cycles were emphasized, for example, the unboundedness of the basin of attraction of the real cycle, in contrast with what happens in the complex case. Finally, plots of the basins of attraction were shown, suggesting an intertwined fractal nature of these basins.

The Matlab and Mathematica codes used to check the attractivity of the coquaternionic cycles and to draw their basins of attraction are available at the site: w3.math.uminho.pt/Maplt. 


\section{Acknowledgments}

Research at CMAT was financed by Portuguese Funds through FCT - Fundação para a Ciência e a Tecnologia, within the Project UID/MAT/00013/2013. Research at NIPE has been carried out within the funding with COMPETE reference number POCI-010145-FEDER-006683, with the FCT/MEC's (Fundação para a Ciência e a Tecnologia, I.P.) financial support through national funding and by the ERDF through the Operational Programme on "Competitiveness and Internationalization - COMPETE 2020" under the PT2020 Partnership Agreement.

\section{References}

[1] Y. Alagöz, K.H. Oral, and S. Yüce. Split quaternion matrices. Miskolc Math. Notes, 13:223-232, 2012.

[2] E. Ata and Y. Yayli. Split quaternions and semi-Euclidean projective spaces. Chaos, Solitons \& Fractals, 41(4):1910-1915, 2009.

[3] B. Aulbach. Continuous and Discrete Dynamics Near Manifolds of Equilibria, volume 1058 of Lecture Notes in Mathematics. Springer-Verlag, Berlin, 1984.

[4] A.N. Baptista, C.C. Ramos, and N. Martins. Iteration of quadratic maps on matrix algebras. Int. J. Bifurcation and Chaos, 22(06):1250150-1-1250250-7, 2012.

[5] A.F. Beardon. Iteration of Rational Functions: Complex Analytic Dynamical Systems. Graduate Texts in Mathematics. Springer-Verlag, 2000.

[6] S. Bedding and K. Briggs. Iteration of quaternion maps. Int. J. Bifurcation and Chaos, 5:877-881, 1995.

[7] S. Bedding and K. Briggs. Iteration of quaternion functions. Amer. Math. Monthly, 103:654-664, 1996.

[8] D.C. Brody and E.-M. Graefe. On complexified mechanics and coquaternions. J. Phys.A: Math. Theory, 44:1-9, 2011.

[9] W. Buchanan, J. Gomatam, and B. Steves. Generalized Mandelbrot sets for meromorphic complex and quaternionic maps. Int. J. Bifurcation and Chaos, 12(8):1755-1777, 2002.

[10] J. Cockle. On systems of algebra involving more than one imaginary; and on equations of the fifth degree. Philos. Mag., 35(3), 434-435, 1849.

[11] M.I. Falcão, F. Miranda, R. Severino, and M.J. Soares. Polynomials over quaternions and coquaternions: a unified approach. In: Gervasi, 0., Murgante, B., Misra, S., Borruso, G., Torre, C.M., Rocha, A.M.A.C., Taniar, D., Apduhan, B.O., Stankova, E., Cuzzocrea, A. (eds.), ICCSA 2017. LNCS, vol. 10405, pp. 379-393. Springer, Cham, 2017.

[12] P. E. Fishback. Quadratic dynamics in binary number systems. J. Difference Equ. Appl., 11(7):597-603, 2005.

[13] P. E. Fishback and M. D. Horton Quadratic dynamics in matrix rings: tales of ternary number systems. Fractals, 13(2):147-156, 2005.

[14] I. Frenkel and M. Libine. Split quaternionic analysis and separation of the series for $S L(2, \mathbb{R})$ and $S L(2, \mathbb{C}) / S L(2, \mathbb{R})$. Adv. Math., 228(2):678-763, 2011.

[15] R. Galeeva and A. Verjovsky. Quaternion dynamics and fractals in $\mathbb{R}^{4}$. Int. J. Bifurcation and Chaos, 09(09):1771-1775, 1999.

[16] C. Gao, X. Chen, Y.-G. Shen. Quintessence and phantom emerging from the split-complex field and the split-quaternion field. Gen. Relativ. Gravit., 48:11, 2016.

[17] M. Gogberashvili. Split quaternions and particles in (2+1)-space. Eur. Phys. J. C, 74:3200, 2014. 
[18] J. Gomatam, J. Doyle, B. Steves, and I. McFarlane. Generalization of the Mandelbrot set: quaternionic quadratic maps. Chaos Solitons \& Fractals, 5:971-986, 1995.

[19] W.R. Hamilton. A new species of imaginaries quantities connected with a theory of quaternions. Proceedings of the Royal Irish Academy, 2:424-434, 1843.

[20] J.A.R. Holbrook. Quaternionic Fatou-Julia sets. Ann. Sci. Math. Québec, 11(1):79-94, 1987.

[21] L. Kula and Y. Yayli. Split quaternions and rotations in semi Euclidean space $E_{2}^{4}$. J. Korean Math. Soc., 44:1313-1327, 2007.

[22] M. Lakner, M. Ǎkapin-Rugelj, and P. Petek. Symbolic dynamics in investigation of quaternionic Julia sets. Chaos Solitons \& Fractals, 24(5):1189 - 1201, 2005.

[23] H.R. Malonek. Quaternions in applied sciences. A historical perspective of a mathematical concept. 17th Inter. Conf. on the Appl. of Computer Science and Mathematics on Architecture and Civil Engineering, Weimar, 2003.

[24] B.B. Mandelbrot. The Fractal Geometry of Nature. W.H.Freeman, San Francisco, California, 1982.

[25] J. Milnor. Dynamics in One Complex Variable. Annals of Mathematics Studies. Princeton University Press, Princeton, 2011.

[26] S. Nakane. Dynamics of a family of quadratic maps in the quaternion space. Int. J. Bifurcation and Chaos, 15(08):2535-2543, 2005.

[27] M. Özdemir and A.A. Ergin. Some geometric applications of split quaternions. In Proc. 16th Int. Conf. Jangjeon Math. Soc., volume 16, pages 108-115, 2005.

[28] M. Özdemir and A.A. Ergin. Rotations with unit timelike quaternions in Minkowski 3-space. J. Geometry Phys., 56(2):322 - 336, 2006.

[29] H.-O. Peitgen, H. Jurgens and D. Saupe. Chaos and Fractals, New Frontiers of Science. Springer-Verlag, New-York, 1992.

[30] P. Petek. On the quaternionic Julia sets. In T. Bountis, editor, Chaotic Dynamics: Theory and Practice, pages 53-58. Springer US, Boston, MA, 1992.

[31] P. Petek. Circles and periodic points in quaternionic Julia sets. Open Syst. Inf. Dyn., 4(4):487-492, 1997.

[32] A.K. Serenevy. Dynamics of polynomial maps of $2 \times 2$ real matrices. In Proc. 16th Summer Conf. Genral Topology and Its Applications, pages 763-778, 2001/2002. 